\title{
A Statistical Model for Optimizing Output
}

\author{
A new statistical model that optimizes a system's output could help to \\ maximize the efficiency of mechanical systems. \\ By Erika K. Carlson
}

W ith a mechanical system like an engine or even a power grid, it pays to know how hard you should push the system to maximize output without it breaking down. Driving the system harder might increase its output but it can make breakdowns more frequent and repairs more costly. Now, Sid Redner of the Santa Fe Institute in New Mexico and colleagues have designed a model to optimize the output of a system using a mechanism that they call first-passage resetting.

Researchers have previously used resetting mechanisms in stochastic search processes. A typical example models the search as a particle on a random walk. Returning the particle regularly to its starting place to begin its random walk anew can make the search more efficient.

Redner and colleagues apply their new first-passage resetting mechanism to an optimization problem. In their model, a particle's location in its random walk represents how hard a system is being driven. If the particle reaches some threshold representing the point at which the system breaks down, it is

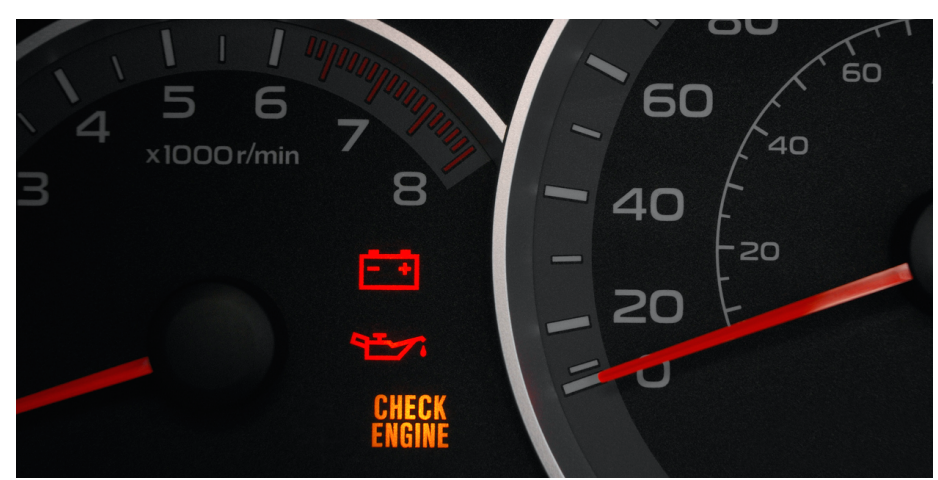

Credit: GarysFRP/iStock/Getty Images reset to its origin. The researchers drive the particle so that it is more likely to spend time near this threshold, describing a system that's working close to its capacity. They therefore find the optimal driving level that maximizes the performance of this idealized system.

The work is theoretical, and though it's too early to apply it to systems as complex as power grids, the researchers say it may be able to offer some insights into the efficiency of car and airplane engines. With further developments, the model could have applications in a wide range of areas, from commerce to population genetics.

This research is published in Physical Review Letters.

Erika K. Carlson is a Corresponding Editor for Physics based in New York City. 